\title{
La santé globale : biopolitique en territoires
}

\section{Alain Vaguet}

\section{OpenEdition \\ Journals}

Édition électronique

URL : http://journals.openedition.org/rfst/726

DOI : $10.4000 /$ rfst.726

ISSN : 2492-3672

Éditeur

Espaces et SOciétés (UMR 6590)

\section{Référence électronique}

Alain Vaguet, "La santé globale : biopolitique en territoires », Revue francophone sur la santé et les

territoires [En ligne], Miscellanées, mis en ligne le 17 septembre 2018, consulté le 06 avril 2021. URL http://journals.openedition.org/rfst/726 ; DOl : https://doi.org/10.4000/rfst.726

Ce document a été généré automatiquement le 6 avril 2021.

\section{cc) (†) (2)}

La Revue francophone sur la santé et les territoires est mise à disposition selon les termes de la Licence Creative Commons Attribution - Pas d'Utilisation Commerciale - Partage dans les Mêmes Conditions 4.0 International. 


\title{
La santé globale : biopolitique en territoires
}

\author{
Alain Vaguet
}

1 La santé dans le monde est devenue un objet de recherches, abordé par la plupart des disciplines des Sciences de l'Homme et de la Société. Ces travaux traitent souvent des conséquences de la mondialisation contemporaine sur le secteur de la santé. Les géographes se sont, bien entendu, longuement penchés sur les "questions d'échelles » (Landy, 2002), cependant ils ont moins souvent que les autres, systématisé le recours au terme santé mondiale ou globale. Cependant, le tout premier chapitre d'un manuel traitant spécifiquement des "Géographies de la santé globale » vient d'être publié par Brown (2018). Il y évoque la géo-histoire des principaux épisodes de cette globalisation, expose ses divers temps et postures "colonialistes/tropicalistes", dénonce le néo libéralisme globalisé et surtout, il lance un appel à l'examen critique des réponses qui sont apportées actuellement aux problèmes posés à la santé dans le monde.

2 C'est précisément ce genre de chantier que nous souhaitons encourager dans cet article, avec la volonté de faire partager à toutes les disciplines, l'apport de la lecture géographique en santé globale. Si la première partie du texte consiste effectivement, à susciter le développement de ces aspects sous l'angle des lieux et des territoires, le second propose une introduction à la question des médicaments, un objet de recherche à penser globalement.

3 Travaillant avec un petit groupe de doctorants sur la mondialisation et la santé en Inde (Vaguet et al., 2009), ces questions, qui pouvaient sembler exotiques, nous ont insensiblement conduit vers les enjeux de la santé globale, au fur et à mesure que ce pays devenait la "pharmacie du monde». En la matière, la période contemporaine, pourtant réputée ouverte aux réseaux et flux, a secrété de redoutables effets barrières, qui créent des conditions d'accès inégales. 


\section{Qu'est-ce qui rend la santé globale?}

\section{De l'internationalisation à la mondialisation}

4 Pour avoir précisé très tôt l'importante transition entre les époques d'internationalisation et celle de la mondialisation, l'article le plus souvent cité dans le domaine, vient du Département de Politique de Santé Publique à la London School of Tropical Medicine (Walt, 1998). Pour cette auteure, la situation de coopération entre les nations pour des raisons de santé, inaugurée par la Réunion de Paris en 1851, pouvait alors légitimement se nommer santé internationale, car elle concernait l'échelle des nations et l'organisation des interactions entre elles. Avec les dispositions mise en place après la seconde guerre mondiale par les agences intergouvernementales des Nations Unies, les préoccupations commencèrent à se mondialiser car non seulement elles s'universalisaient mais elles prenaient, peu à peu, une certaine distance vis-à-vis des seuls Etats. On pourrait résumer cette situation dans le vocabulaire des sciences de la complexité, en disant qu'à partir de ce moment, la santé du monde ne se résume plus à la somme des interactions entre les Etats (les risques liés aux relations internationales, relatives aux faits de santé) depuis qu'émergent des phénomènes mondiaux ou globaux, parfois voulus (libéralisation/ privatisation) mais pas toujours (croissance des inégalités).

\section{La biopolitique globale}

5 Les chercheurs anglophones utilisent régulièrement le terme global pour caractériser la santé dans le monde. En français, à l'inverse, ce terme global pose quelques problèmes car il «reçoit une multiplicité d'acceptions » (M. Reghazza, 2015). On peut rappeler que dans certains cas et pour certains auteurs, le terme global doit remplacer celui de mondial, du fait des mutations récentes du capitalisme, qui disputent aux Etats leurs anciennes prérogatives (C. Gobin, 2012). Pour certains marxistes à l'inverse, il n'apporte rien de plus au terme mondial (R.J. Johnston, 2009). Pour notre part, nous utiliserons indistinctement les termes, global, mondial et planétaire, acceptant la catégorie santé globale en tant qu'intitulé rassembleur et multilingue.

Mais tout cela est-il légitime? N'est-ce pas simplement la création d'une niche éditoriale qui empiète sur les publications et enseignements bien établis, comme ceux relatifs aux relations internationales ou à la géopolitique? Bozorgmehr (2010) a cherché à clarifier cette question, ce qui l'a amené à essayer de définir finement ce qui est global et ce qui ne l'est pas vraiment. Au final, pour lui « le global en tant que supra territorial se restreint aux liens entre les processus par lesquels toute personne, n'importe où dans le monde, est contrainte ou victime ou résistante à des forces extérieures ». On voit bien l'idée mais pour les géographes et les sociologues, il reste que le supra territorial est encore en territoires. On peut trouver une bonne armature théorique qui épaule l'idée de Bozorgmehr, en faisant référence au terme biopolitique (Foucault, 1979). Il qualifie une forme d'exercice du pouvoir sur les territoires et ce faisant, sur la vie des individus et des populations. La biopolitique peut facilement caractériser l'évolution néo libérale des politiques de santé publique et la montée de l'individualisation du risque dans les dispositifs de santé publique. Ce terme nous offre 
ainsi un espace intellectuel large pour observer la domination et le contrôle socioterritorial, développés par les autorités politiques (T. Bossy, F. Briatte ; 2011).

\section{La crise de la santé publique complexifie la gouvernance}

7 Les urgences contemporaines de la santé publique des trente dernières années ont défié tous les niveaux infra ou supra territoriaux, dans la mesure où ils semblent tous avoir été pris en défaut par le retour des risques infectieux, notamment par le sida, la grippe et le virus Ebola. Alors que l'espérance de vie sur la planète s'améliorait, la succession de ces sinistres épisodes, a engagé une crise de confiance des citoyens à l'égard des sciences de la santé et surtout une crise de légitimité des instances mondiales (Organisation Mondiale de la Santé) dans lesquelles de nouveaux venus (la Banque Mondiale, le Fonds mondial, des Fondations...) se sont ajoutés, complexifiant la gouvernance de la santé dans le monde. Des revues en ligne, comme par exemple "Globalization and Health ${ }^{1}$ " ont rapidement pris conscience de l'effervescence qui régnait autour des "dimensions globales de la santé internationale». Ils ont donc cherché à capter les articles traitant de l'économie politique des échanges internationaux relatifs à la santé, des migrations des professionnels de la santé, du tourisme médical, des réformes parfois convergentes des systèmes de santé, de la santé des migrants, de la diplomatie de la santé... Tous ces objets pour lesquels, la dimension critique souhaitée par Brown reste à développer en géographie, au profit de toutes les disciplines.

\section{Pour une «Philanthropolitique » critique, de la santé}

8 En pratique, la réaction aux urgences sanitaires récentes s'est construite autour d'une critique sans nuance de la bureaucratie des agences onusiennes mais également, de l'inefficacité des gouvernements des pays les plus pauvres, réputés incapables d'utiliser les fonds d'aide au développement pour endiguer les risques ${ }^{2}$. De nouvelles pratiques, portées par la vague politique néo-libérale, ont alors consisté à contourner les pouvoirs nationaux et multilatéraux, au profit de nouveaux types de gouvernance. Le Fonds Mondial $^{3}$, en accord avec les philanthropes a ainsi focalisé ses actions contre une sélection de maladies transmissibles, même si la mortalité par diabète ou diarrhées infantiles demeure supérieure et si le curatif fait oublier la prévention. La libéralisation économique et en particulier l'ajustement structurel ${ }^{4}$, ont porté la privatisation des systèmes de soins, dans le but de les rendre plus efficaces et rentables. Tous les acteurs ont ainsi emboité le pas de la Banque Mondiale, convertie à partir de 1993 à l'investissement dans la santé, «condition nécessaire à la prospérité économique ». A partir de l'an 2000, la Banque Mondiale est devenue premier financeur des programmes de santé dans le monde. Ce faisant, le secteur de la santé passait du vocabulaire de l'humanitaire à celui de l'entreprise, centré soudainement sur la terminologie du coût/ efficacité des actions, des programmes de Participation Public/Privé (PPP) et à la responsabilisation des individus devant payer eux-mêmes pour leurs soins. En somme, moins d'Etat et plus de responsabilité individuelle.

9 La recherche de nouveaux financements pour les questions de santé globale, fait souvent passer au second plan les débats sur les stratégies. Ainsi, les grands programmes verticaux contre les maladies transmissibles (type Fonds Mondial), rassemblent des budgets très conséquents dont les résultats immédiats indéniables 
cachent les difficultés de financement à long terme. L'éradication éventuelle demeurant encore fort éloignée dans le temps, les Etats ont rapidement pris le relais des fonds privés (Amat et Vaguet, 2012). Par ailleurs, certains auteurs, jugent que la verticalité s'est organisée au détriment de la mise en place de la santé primaire et de la prévention (Birn, 2009, Packard, 2016). Ils regrettent que les options thérapeutiques soient systématiquement focalisées vers les moyens techniques sophistiqués : nouveaux médicaments et vaccins ; ils contestent ces décisions qui tendent toujours à favoriser la mise en place de marchés profitables. Au total, on parle désormais du «capitalisme pharmaceutique " (Quet, 2017), axé sur la rentabilité et son incapacité à mettre les fruits des recherches au service de tous. En somme, on en revient à l'opposition entre la liberté, d'entreprendre, d'aider, de choisir, face au principe de justice et de partage, notamment lorsque des effets barrières (brevets) paralysent l'accès aux substances essentielles (ARV contre le sida). Cette question des médicaments fait l'objet de la partie suivante.

\section{L'inégalité d'accès aux médicaments : un déterminant de santé globale}

10 Les produits pharmaceutiques essentiels constituent des indicateurs précieux de la santé publique globale, du fait de leur disparité d'accès dans le monde. Aucune stratégie viable n'a pour le moment été en mesure de réduire les déséquilibres mondiaux. La recomposition territoriale de leur production, c'est à dire l'irruption des producteurs des Suds (Inde, Chine, par exemple), constitue pourtant un basculement historique "contre hégémonique ». Comme pour beaucoup d'autres marchandises (tee shirts, électronique...) les flux pharmaceutiques Sud-Sud et Sud-Nord auraient pu nettement réduire les prix. Les pays "émergents" ont en effet bouleversé tous les anciens repères avec les génériques bon marché mais les règles internationales (Droits de Propriété Intellectuelles DPI, en 1995) et la recherche du profit au détriment de la santé publique, en limitent la portée dans tous les territoires. Les cartes sont difficiles à établir faute de données mais de grandes zones restent encore hors de portée des médicaments essentiels, sans même parler des innovations. Les médicaments restent donc chers et même hors de prix pour certaines spécialités nouvelles (VIH, hépatite $\mathrm{C}$, cancers...). Dans le même temps, d'autres substances anciennes (vaccins, antibiotiques...) n'intéressent déjà plus les producteurs du monde entier, provoquant des tensions d'approvisionnement et ruptures de stocks inédites. En France, on recense désormais entre 400 et 500 signalements de "tensions » par an sur le site de l'Agence Nationale de Sécurité des Médicaments (ANSM). Un symptôme assez récent (dans les pays du Nord), qui laisse à penser que le système complexe du médicament, pourtant relativement encadré, nécessite des améliorations pensées en terme globaux.

11 Pour présenter rapidement ces enjeux, on peut dire que les acteurs les plus attendus en matière de réformes destinées à faciliter l'accès, comme les Etats et les organismes multilatéraux, montrent une certaine faiblesse et restent prisonniers de leurs contradictions : ils ne protègent pas efficacement les populations en laissant prospérer, lobbies, marchés principaux et parallèles, au risque des contrebandes qui se mettent en place pour compenser (mal) les inégalités d'accès. Au final, les acteurs les plus puissants demeurent donc les producteurs et face à eux, on ne trouve guère que des organisations de citoyens globaux pour représenter les intérêts des malades (MSF, 
Oxfam, Network of Positif People...). Quels arguments avancent les diverses parties prenantes?

\section{Logique du producteur et logique de l'utilisateur}

12 A partir des points de vue exprimés lors de la Journée de l'association Remed ${ }^{5}$, Paris, 2016, examinons la logique des producteurs ${ }^{6}$ représentés par le syndicat professionnel : Les Entreprises du Médicament (LEEM). Face aux militants (MdM, MSF) qui leur reprochent une hauteur des prix qui finance plus le marketing que la recherche, ils défendent le secret des affaires et les prix élevés qui, dans leur esprit, permettent de financer une recherche d'excellence, pour produire des médicaments de haute qualité. Il est vrai que pour un principe actif autorisé, des milliers de tentatives sont infructueuses et qu'il faut financer les essais cliniques. Cependant, les fonds publics sont aussi mis à contribution pour la recherche. Des citoyens américains et anglais notamment (Global Justice Today, 2017), protestent régulièrement car lorsqu'ils achètent leurs médicaments chers, ils estiment qu'ils paient deux fois : ils considèrent en effet, qu'ils ont déjà contribué à financer une partie des recherches avec leurs impôts. Aux Etats Unis : «13,6 milliards de dollars de financement de la recherche fondamentale est fournie par le National Institutes of Health (NIH financé par les contribuables). En revanche, l'industrie n'aurait comparativement consacré que 6,3 milliards de dollars à la recherche fondamentale en $2014 »^{7}$. En pratique, impatients d'augmenter leurs recettes, certains fabricants ont changé leur approche de fixation des prix, en se basant moins sur le coût de revient des nouveaux produits que sur la propension à payer des acheteurs. Sans aller aussi loin, beaucoup d'industriels et d'Etats, se contentent assez efficacement de mettre des barrières à l'entrée des génériques sur le marché, pour limiter la concurrence et ainsi continuer de pratiquer des prix élevés. Une ancienne spécialité, comme l'insuline par exemple, dont l'exclusivité du brevet est tombé depuis longtemps, a vu son prix s'emballer (Kaplan, 2013). Point fort de leur argumentaire, les fabricants du LEEM avancent que $95 \%$ des médicaments ne sont plus sous licence. Sous-entendu, ils peuvent être copiés par un producteur générique, preuve selon eux, que le prix n'est pas la barrière principale à l'accès.

Pour expliquer ce chiffre de $95 \%$ il faut rappeler que tous les médicaments ne sont pas essentiels et qu'une bonne part d'entre eux résulte de stratégies commerciales. Une vive polémique est engagée, depuis longtemps, contre la Némésis et la "pharmaceuticalisation» de la vie (Nichter 1996); autrement dit, contre la dérive mercantile de l'industrie pharmaceutique, qui cherche à multiplier les innovations, au risque des dérives et des scandales à répétition.

\section{Un « système complexe global »}

14 En résumé, comme le montre la figure simplifiée (ci dessous) et ses liens relationnels, inspirés des systèmes dynamiques (Bigdeli, 2012); ce que l'on peut nommer le "système complexe des médicaments " se trouve face à un dilemme classique en analyse spatiale : une alternative entre deux logiques, celle des producteurs ou celles des utilisateurs. 
Les industriels du médicament cherchent à fabriquer à bas coûts. Pour ce faire, ils assemblent des produits de base, importés des pays intermédiaires. En revanche, ils vendent à des prix élevés, protégés par les accords du commerce mondial (OMC), dont le très débattu Droit de Propriété Intellectuelle (DPI). Ceci au dépend de l'accès des utilisateurs et des organisations collectives qui assurent les remboursements de frais de santé. De leurs côtés, les Etats et les Organisations Inter Gouvernementales (OIG), censés protéger la santé des citoyens dans le cadre des droits de l'homme et des droits à la santé, disposent de quelques moyens légaux : la négociation collective, l'utilisation des dispositifs des Licences Obligatoires (LO, une dérogation aux brevets pour cause d'urgence de santé publique). Mais ces institutions utilisent peu ces moyens, qui « violenteraient » les producteurs, par ailleurs créateurs d'emplois et de richesses. Elles préfèrent compter sur de nouvelles solutions Public-Privé, comme l'Initiative pour les vaccins (GAVI) ou Medicines Patent Pool (MPP) etc. Dans cette dernière institution, des négociations permettent d'amener les titulaires de brevets à produire des génériques pour les pays pauvres, contre le sida, la tuberculose et l'hépatite C. Mais l'efficacité de cette pratique reste somme toute limitée, même s'il est question qu'elle s'étende progressivement à toute la liste des médicaments essentiels.

Fondamentalement, ces nouvelles institutions qui tentent de compenser les injustices du système, participent donc à sa prolongation. Une bifurcation majeure pourrait advenir si les débats autour du médicament comme «bien commun» (Cassier 2017) venaient à remettre en questions les brevets décidés en 1995. C'est cependant plutôt leur renforcement qui fait l'actualité.

17 En compensation des inégalités d'accès qu'ils contribuent à créer, les industriels proposent des consolations, comme les cadeaux, sous forme de médicaments pour des pays pauvres, ou des licences volontaires (LV) de leurs propres productions, pour certains pays, ce qui, au passage, permet de bloquer l'arrivée de la forme générique (Londeix 2014) ${ }^{8}$. Face aux critiques persistantes, certains fabricants ont également réagi en faisant varier leurs prix selon une typologie de pays : prix élevés pour les pays des Nords (Etats intermédiaires inclus, là où les patients sont les plus nombreux) et prix réduits pour les pays les moins avancés. Ceci peut donner l'impression de réductions importantes, alors que ces derniers pays restent encore en dehors des Droits de propriété Intellectuelle pour plusieurs années et qu'ils peuvent officiellement se fournir en génériques bon marché. Dans ce contexte, somme toute bien stable, tout juste commence-t-on à discuter d'options à l'OMS, par exemple autour du juste prix des médicaments qui pourrait satisfaire tous les partis (Rapport Fair price, OMS, 2017). Pendant ce temps, les industriels (des Nords et des Suds) se désengagent de certains segments de production qui ne sont pas avantageux et qui connaissent dès lors les ruptures de stocks : symptôme d'un système des médicaments, plus guidé par l'intérêt que par des considérations de santé publique.

18 Au final, le système complexe du médicament est entré dans une dimension planétaire, à la fois pour la production, la consommation, et la régulation mais aussi par toute une série d'actions et de rétroactions qui s'équilibrent, au dépend des utilisateurs. Au final, l'industrie pharmaceutique demeure (très) rentable et les inégalités d'accès perdurent. Pour contredire les célèbres travaux de Hardin (1968), on pourrait parler pour les médicaments de la tragédie de l'inaccessibilité. 
Figure : Le système complexe des médicaments ou la tragédie de l'inaccessibilité.

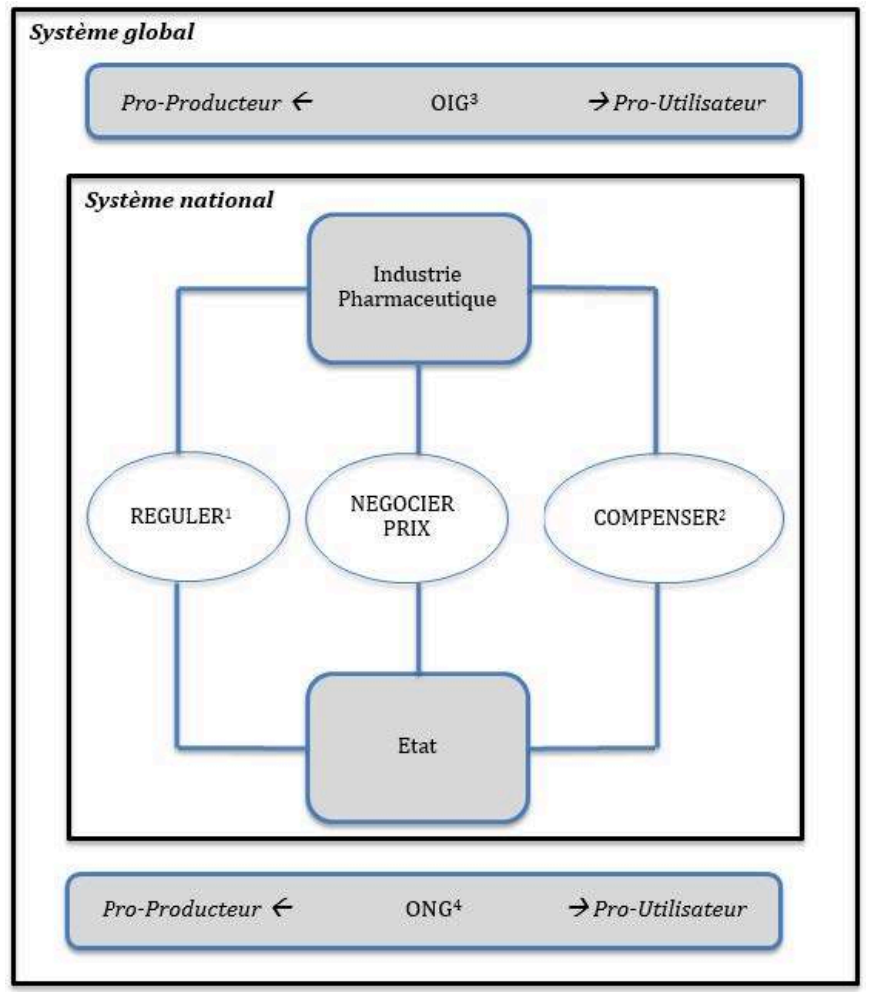

1. OUTILS DE RÉguLATION : Autorisation de Mise sur le Marché (AMM) ; Droits de propriété intellectuelle (DPI) ; Licence Obligatoire (LO) = permis de copier attribué aux Etats pour raison de santé publique ; - 2. OUTILS DE COMPENSATION : Licence Volontaire $(\mathrm{LV})=$ permis de copier octroyé par les industriels = pour éviter les génériques ? Offrir des « cadeaux »; MPP : Medicines Patent Pool - 3. olG PRO-PRODucteur : ex : I'OMC en défendant le Droit de Propriété Intellectuelle; OIG Pro-Utilisateur : I'OMS en défendant, le de-linkage (ne plus lier le prix des spécialités aux coûts de recherche) ou le Juste Prix , soit un prix acceptable pour les fabricants et pour les utilisateurs («Fair Price ») ; I'ONU en défendant les Droits de la santé à travers les Droits de l'Homme - 4. ONG PRO-PRODUCTEUR : ex : les fondations (Bill Gates) ; ONG Pro-Utilisateur : ex : MSF, Oxfam...

\section{Discussion/conclusion}

Les configurations sanitaires biopolitiques globales, très rapidement évoquées dans ce texte, montrent comment de nouvelles formes de pouvoir s'exercent, produisent et maintiennent des inégalités dans l'espace-mondialisé. Un thème fécond de recherche en Sciences de l'Homme et de la Société (SHS) s'ouvre, autour de la santé globale et en particulier de sa gouvernance (Global Health Governance). Une "gouvernementalité " renouvelée qui émerge depuis la fin du $\mathrm{XX}^{\mathrm{ème}}$ siècle, pour le meilleur ou pour le pire, en particulier avec la montée en puissance de la philanthropie dans ce domaine. Un phénomène souvent accueilli avec bienveillance, lorsqu'il concerne l'amélioration de la santé des populations les plus démunies mais dont l'examen critique contribue à dévoiler un autre visage du leadership américain sur le destin du monde et de sa santé. Beaucoup d'auteurs, politistes en particulier, comme Dixneuf (2003), ont mis en avant les raisons qui poussent une grande puissance à se mêler des questions de santé dans les Suds, en particulier pour des raisons géostratégiques et commerciales. BerthoHuidal (2012) a démontré que ce phénomène géopolitique était situé, Il prolonge et amplifie la tradition américaine, blanche et anglo saxonne de charité, qui est imaginée 
comme un rempart au multiculturalisme. Les historiens, psychologues, économistes, ont conduit de passionnantes recherches sur l'envers du décor de la philanthropie, ils montrent parfaitement les ressorts qui agissent dans l'arrière-cour de la générosité. Certains, comme Packard (2016), en arrivent à condamner la prétention des spectaculaires bonnes actions qui ne cherchent ni à s'attaquer aux racines des maux contemporains d'inégalités de la santé globale, ni à leur dérives marchandes.

Finalement, les logiques opposées des constructeurs et des utilisateurs semblent parfaitement résister au passage du temps. Les alternatives - comme l'idée de replacer les médicaments en tant que "bien commun" pour qu'ils échappent aux jeux mercantiles et redeviennent plus accessibles-restent pour l'instant théoriques,. Pourtant, les injustices semblent criantes, un seul exemple saisissant : sur 7 milliards de terriens, l'OMS en comptabilise plus de 5,5 milliards qui n'ont pas accès aux substances anti douleur et la situation n'évolue guère. On peut voir par-là quelques conséquences $\mathrm{du}$ «capitalisme financiarisé ou logistique» (Quet, 2017) qui régit le monde du médicament. Pour autant, très pragmatiquement et en réaction à la hauteur des prix, aux rigidités des brevets et des univers réglementaires, des nouveaux écosystèmes de production se mettent en place aux Etats Unis et ailleurs, sur la base du «Do It Yourself ». Par exemple, des communautés de chercheurs "hackeurs » initient des protocoles de fabrication ouverts, qui contournent les brevets et qui permettent de produire des médicaments «maison» (Gallegos et al., 2018). A ceux qui se scandalisent des risques induits pour la santé publique, ils répondent que les produits pharmaceutiques inabordables constituent un danger encore plus grand.

21 Au total, l'ensemble des processus et des acteurs en jeux, institutionnels ou non, qui gèrent les corps et la santé des contemporains, constitue désormais une nébuleuse complexe et globale, manquant souvent de transparence pour être bien comprise. Elle nécessite une attention soutenue des chercheurs qui souhaitent promouvoir le droit à la santé. Avec les plus motivés, comme le géographe Brown (2018) ou l'anthropologue Appaduraï (2013), il nous semble important d'appeler les sciences sociales à développer des travaux critiques à l'échelle planétaire, dans un objectif de citoyenneté globale.

\section{BIBLIOGRAPHIE}

Amat-Roze J.M., Vaguet A., 2012, Qui gouverne la santé du monde ? In Dix défis pour la planète (Dubresson et Veyret Ed.), Editions Autrement.

Appadurai A. 2013, Condition de l'homme global, Payot, $421 \mathrm{p}$.

Bertho Huidal M., 2012, « Charité business » le grand marché de la santé mondiale, Vedémiaire.

Bigdeli M., Jacobs B., Tomson G., Laing R., Ghaffar A., Dujardin B., Van Damme W., 2013, Access to medicines from a health system perspective. Health Policy and Planning $\mathrm{n}^{\circ} 28$.

Bossy T., Briatte F, 2011, les formes contemporaines de la biopolitique, in Revue Internationale de Politique Comparée, vol 18, $n^{\circ} 4$. 
Birn A.E., Pillay Y., Holtz T., 2009, Textbook of International Health. Oxford University Press. Brown T., 2018, Global health geographies, in Brown T., Andrews G. J., Cummins S., Greenhough B., Lewis D., Power A., Health geographies: a critical introduction, Wiley - Blackwell.

Bozorgmehr K., 2010, Rethinking the 'global' in global health : a dialectic approach. Globalization and Health, vol 6, $\mathrm{n}^{\circ}$ 1, http://www.globalizationandhealth.com/content/6/1/19 (consulté en juin 2017)

Cassier M., 2017, Médicament et communs, in Cornu M. and al. (édit) Dictionnaire des biens communs, Quadrige Dico Poche.

Dixneuf M., 2003, la santé, enjeu de la gouvernance mondiale. Etudes du CERI, $n^{\circ} 99$.

Foucault M., 1979, Naissance de la biopolitique, Cours au Collège de France, publiés en 2004, éditions Gallimard.

Gallegos E.J., Boyer C., Pauwels E., Kaplan W., Peccoud J., 2018, The open insuline project : a case study for bio « hacked » medicines, Trends in Biotechnology (TIBTECH 1681) DOI:https://doi.org/ 10.1016/j.tibtech.2018.07.009.

Global Justice Today and Stop Aids, 2017, Pills and profits: How drug companies make a killing out of public research. https://stopaids.org.uk/wp/wp-content/uploads/2017/10/Pills-andprofits-report-WEB-002.pdf (consulté en janvier 2017)

Ghorra Gobin C., 2012 Dictionnaire de la mondialisation, Armand Colin, 645 p.

Hardin G., 1968, The Tragedy of the Commons. Science, vol. 162. n 3859, p. 1243-1248 Site de Science [archive] (consulté en juin 2018)

Johnston, R.J., 2009, Dictionnary of Human geography, Wiley, Blackwell, 1052 p.

Kaplan W. A., Beall R.F., 2013, The global intellectual property ecosystem for insulin and its public health implications: an observational study. J. Pharm. Policy Pract. 2017 ; 10:3. PMID: 27446593.

Landy F., (Dir.), 2002, De la mondialisation au développement local en Inde : Questions d'échelles, CNRS Editions, $254 \mathrm{p}$.

Les Entreprises du Médicament (LEEM) Consultation 2017 http://www.leem.org

Nichter, M. and M., 1996, Anthropology and International Health: Asian Case Studies. New York: Gordon and Breach Publishers.

Packard R. M., 2016, A history of global health, interventions into the lives of other peoples, Johns Hopkins University Press, 414 p.

Quet M., 2017, Pharmaceutical capitalism and its logistics: access to hepatitis C treatment, Theory, Culture and Society, Sage Journal, https://doi.org/10.1177/0263276417727058 (consulté en Mars 2018).

M. Reghazza, 2015, De l'avénement du monde à celui de la planète, Habilitation à Diriger des Recherches, https://tel.archives-ouvertes.fr/tel-01255031/document (Consulté en septembre 2018).

Vaguet A. (ed), 2007, Indian health landscapes under globalization, Manohar Publishers, 350 p.

Walt G., 1998, Globalization of international health, The Lancet, vol. 351, Issue 9100. 


\section{NOTES}

1. https://globalizationandhealth.biomedcentral.com/

2. On rejoint ici encore une idée de Foucault, utilisée par Reghezza (2015), selon laquelle « le risque est un dispositif au fondement de la gouvernementalité ».

3. Cette institution crée en 2002, récolte des fonds, venus surtout des Etats mais aussi des Fondations, pour financer la lutte contre le sida, la tuberculose et la malaria dans le monde.

4. Les prêts du FMI ou la Banque Mondiale aux pays en crise économique, sont accordés sous conditions de réformes structurelles, comme la privatisation des secteurs de l'éducation ou de la santé.

5. Réseau Médicaments et Développement (REMED), est une association française, elle anime un forum en ligne et travaille à l'amélioration de l'accès aux médicaments dans les pays en développement.

6. Journées de l'association Remed, table ronde, Paris, 2016

7. The U.S. Government Accountability Office (GAO). Report, conducted on request of U.S. Sen. Bernie Sanders (I-Vt.) and U.S. Rep. Elijah Cummings (D-Md.) about pharmaceutical research and development (R\&D) and business trends). Consulté en 2017 : https://www.citizen.org/media/press-releases/gao-report-undercutspharma-rd-claims

8. Coalition internationale pour la préparation aux traitements en Afrique du Nord et au Moyen-Orient (ITPC-MENA)

\section{RÉSUMÉS}

L'ampleur du processus de mondialisation contemporain ajoute un niveau supplémentaire au tableau des déterminants de la santé. Conséquence principale, cette nouvelle échelle replace l'autorité des Etats dans un complexe décisionnel qui associe les organismes mondiaux, les acteurs de la société civile globale mais aussi les puissants lobbies professionnels et les philanthropes. Ces changements de gouvernance et de financement de la santé globale semblent difficiles à évaluer. Les uns encensent des actions spectaculaires, les autres ne voient que leurs limites. Pour autant, tous les observateurs s'accordent pour regretter la montée des inégalités sanitaires qui résultent des transformations socio-politiques mondiales.

Poursuivant un objectif synthétique, ce texte commence par discuter la pertinence du terme global. Ensuite, prenant l'exemple de l'inégalité d'accessibilité aux médicaments essentiels, il discute la durabilité des brevets d'invention. Finalement il propose d'éclairer les postures incommensurables, adoptées par l'industrie pharmaceutique et les ONG militantes du droit à la santé.

$\mathrm{Au}$ total, le texte vise à convoquer diverses approches pour engager une perspective systémique et ainsi mettre le lecteur sur le chemin de la dimension complexe de la santé globale. 
The 1851 Paris Meeting to build cooperation for health between nations heralded the governance of international health concerning the scale of the nation-state and the organization of the interrelations between them. Following the Second World War, the arrangements put in place through the intergovernmental agencies of the United Nations, enabled an expanded remit of health concerns that became globalized. This new global health governance can be described through the language of the complexity sciences: since the emergence of global phenomena, whether beneficial (liberalization / privatization) or not (growth of inequalities), the influences that shape health conditions across the world are no longer limited to interactions between states.

A good theoretical framework for studying this phenomena can be found by referring to the term biopolitics (Foucault, 1979). It describes a form of exercise of power over territorially defined domains and, in doing so, over the lives of individuals and populations. Biopolitics can easily characterize the neo-liberal evolution of public health policies and the rise of individualisation of risk in public health systems. This term thus offers us a wide intellectual space to observe the domination and the socio-territorial control.

The global drug-scape offers a good example of the dilemma between two dichotomous logics in developing a spatial analysis, that is between a focus on the producers or the users. Drawing on dynamic systems (Bigdeli, 2012), the figure below illustrates some of the relational links.

Pharmaceutical companies are looking to manufacture at low cost. To do this, they assemble commodities imported from intermediate countries. At the same time, they sell at high prices, protected by the World Trade Agreements (WTO) and the controversial agreement on Intellectual Property Rights (IPR). For their part, States and Intergovernmental Organizations (IGOs), supposed to protect the health of the citizens within the framework of human rights and the rights to health, have some legal measures such as: the collective negotiation and the use of Compulsory Licensing ( $C \mathrm{~L}$ is a derogation to patent in the case of a public health emergency). But these institutions make little use of these measures, which would meet opposition from producers who are also creators of jobs and wealth. National governments prefer to rely on new Public-Private solutions, such as Medicines Patent Pool (MPP). These negotiations allow patent holders to produce generic drugs for poor countries, for a limited number of diseases, specifically HIV/AIDS, tuberculosis and hepatitis C. The effectiveness of this practice, however, remains limited, even if the permitted drugs is gradually expanding to the entire list of essential drugs. In conclusion all actors therefore extend and complexify the global drug system, but do not necessarily improve health care justice. A major bifurcation could happen if the debates around the drug as "common good" (Cassier 2017) came to question the IPR decided in 1995. However, it is rather the reinforcement of IPR that makes the news. The global pharmaceutical industries compensate for the inequalities in access to health care to which they contribute through donations of medicines for poor countries, or voluntary licenses (VL) of their own products for certain countries, which, in passing, serve to block the import of generic forms (Londeix 2014). Faced with persistent criticism, some manufacturers have also responded by varying their prices according to a typology of countries: high prices for the countries of the North (intermediate states included, where the patients are the most numerous) and reduced prices for the leastdeveloped countries. This can offer significant reductions but also serves the interests of the pharmaceutical industry while these countries still remain outside the Intellectual Property Rights agreements for a few years, as they could legally supply their needs through importing generic forms.

At present, the system remains stable. But discussions have begun at the WHO for example around the fair pricing of drugs so as to satisfy all parties (Fair price report, WHO, 2017). Meanwhile, manufacturers (from the North and South) are disengaging from certain production segments that are not profitable and that are therefore experiencing stock-outs. Finally, the complex system of the drug has entered a global dimension, both for production, consumption, 
regulation but also by a series of actions and feedback that balance at the expense of users. In the end the pharmaceutical industry remains (very) profitable and inequalities of access persist. To contradict the famous works of Hardin (1968), one could speak for drugs of the tragedy of inaccessibility.

\section{AUTEUR}

\section{ALAIN VAGUET}

Université de Rouen, Maître de conférences, UME 6266 IDEES 